\title{
Ultraviolet spectroscopy of the shell of RR Pic
}

\author{
H. W. Duerbeck \& W. C. Seitter \\ Astronomisches Institut, University of Muenster, F.R.G. \\ M. F. Bode \\ School of Physics \& Astronomy, Lancashire Polytechnic, Preston, U.K.
}

A. Evans

Department of Physics, University of Keele, U. K.

\section{Introduction}

Spectroscopy of the nebular (as opposed to the stellar) remnants of old novae can be a powerful diagnostic of physical conditions in, and mode of excitation of, nova shells. While optical spectroscopy of nova shells has been widely used in this way, few are sufficiently well resolved, and sufficiently bright, to be observed with IUE.

Combined optical and ultraviolet spectroscopy of only two nova remnants-those of GK Per and DQ Her-have been reported to date. Ferland et al. (1984) demonstrated that the shell of DQ Her is extremely cool $\left(T_{e} \simeq 500 \mathrm{~K}\right)$ and is excited by a hot central source. Bode et al. (1988) showed that the ultraviolet and optical lines in the spectrum of the GK Per nebula can best be understood in terms of shock excitation, consistent with its observed properties at radio wavelengths (Seaquist et al. 1989).

We present here a preliminary report of ultraviolet spectroscopy of the shell around RR Pic (Nova Pic 1925). The stellar remnant is a strong ultraviolet source, with a power-law continuum $f_{\lambda} \propto \lambda^{-1.8}$ (Krautter et al. 1981). Optical observations (CCD imaging and spectroscopy) of the nebula are briefly described in Duerbeck (1987).

\section{Ultraviolet spectroscopy}

IUE observations of RR Pic were obtained in 1989 June 18-19. The star was observed at low resolution, through the large aperture, with both SWP and LWP cameras. There is no evidence of an absorption feature around $\lambda 2200$, suggesting that $E(B-V)<0.05 ;$ Krautter et al. (1981) found $E(B-V)=0.01$. For the present we assume that $E(B-V)=0$. The southern equatorial ring region of the nebular remnant was observed through the large aperture of $I U E$ at low resolution, also with SWP (exposure $372 \mathrm{~min}$ ) and LWP (317 $\mathrm{min}$ ) cameras; however little or no signal was obtained in the long wavelength range.

The nebula was detected in the SWP wavelength range. However the stellar remnant is $\sim 10^{\prime \prime}$ away and some scattered light from the star is expected. We have attempted to allow for this using the formulae given by de Boer \& Cassatella (1986). The extracted SWP spectrum, with scattered light from RR Pic itself subtracted, is shown in Fig. 1. Lines of NV $\lambda 1240$, OIV] $\lambda 1400$, NIV] $\lambda 1486$, HeII $\lambda 1640$ and NIII] $\lambda 1750$ are clearly visible. In the original spectrum the CIV $1550 \AA$ feature is present in the spectrum of the nebula; however most of the CIV $\lambda 1549$ line seems to be contributed by scattered light from the star as it is not present in the reduced nebular spectrum (see Fig. 1).

We have compared the relative line strengths with those predicted by shock models (Cox \& Raymond 1985). No agreement with model values is possible, even when the overabundances expected in novae are included. On the other hand, there is good agreement with model nebulae photoionized by power-law radiation continua (Stasińska 1984), provided overabundance of He and $\mathrm{N}$, and underabundances of $\mathrm{O}$ and $\mathrm{C}$, are assumed. 


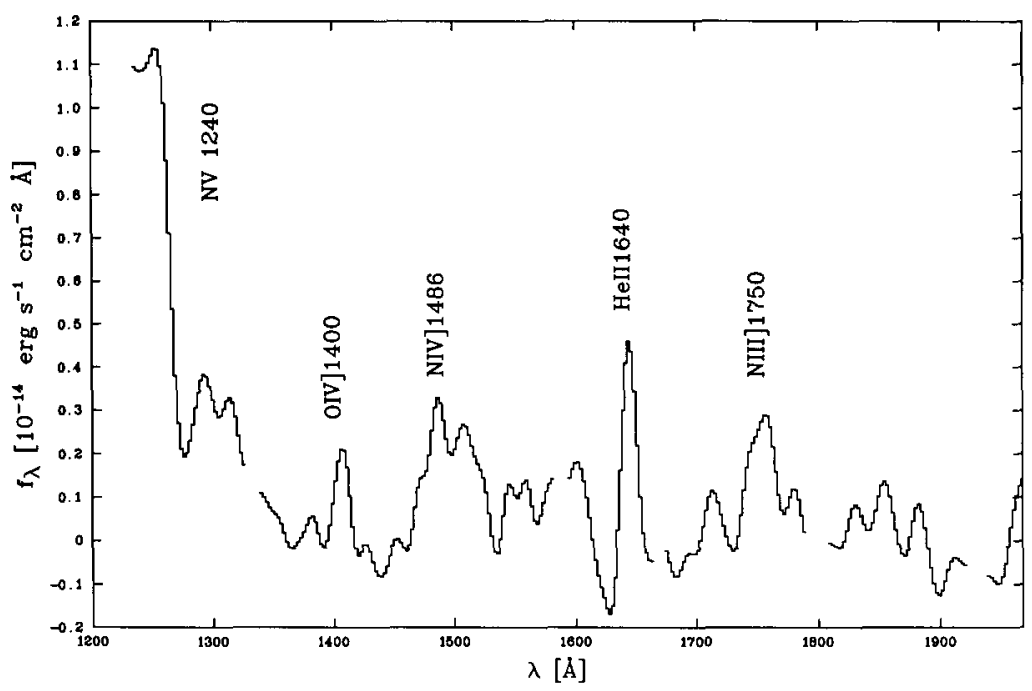

Figure 1: SWP spectrum of the 'southern equatorial ring' around RR Pic.

Optical spectra of the western polar blob region of the nebula are described by Williams \& Gallagher (1979) and Duerbeck (1987). These show lines of [OIII], [NII], [FeV], [FeVI] and [FeVII]. IUE observations of this region are scheduled for 1989 September and will be reported elsewhere.

\section{References}

Bode, M. F., Duerbeck, H. W., Seitter, W. C., Albinson, J. S. \& Evans, A., 1988. In A Decade of Astronomy with IUE, ESA-SP-281, p.183.

de Boer, K. S. \& Cassatella, A., 1986. In New Insights in Astrophysics, ESA-SP-263, p.665.

Cox, D. P. \& Raymond, J. C., 1985. Astrophys. J., 298, 651.

Duerbeck, H. W., 1987. ESO Messenger, 50, 8.

Ferland, G. J., Williams, R. E., Lambert, D. L., Shields, G. A., Slovak, M., Gondhalekhar, P. M. \& Truran, J. W., 1984. Astrophys. J., 281, 194.

Krautter, J., Klare, G., Wolf, B., Duerbeck, H. W., Seitter, W. C., Vogt, N. \& Wargau, W., 1981. Astron. Astrophys., 102, 337.

Seaquist, E. R., Bode, M. F., Frail, D. A., Roberts, J. A., Evans, A. \& Albinson, J. S., 1989. Astrophys. J., in press.

Sparks, W. M., Starrfield, S. \& Truran, J. W., 1978. Astrophys. J., 220, 1063.

Stasinska, G., 1984. Astron. Astrophys. Suppl. Ser., 55, 15.

Williams, R. E. \& Gallagher, J. S., 1979. Astrophys. J., 228, 482. 